\title{
Prevalence of Intestinal Parasitic Infections and Associated Water, Sanitation, and Hygiene Risk Factors among School Children in Mwea Irrigation Scheme, Kirinyaga County, Kenya
}

\author{
Elizabeth Njambi (D), ${ }^{1}$ Dennis Magu, ${ }^{1}$ Janet Masaku, ${ }^{2}$ Collins Okoyo ${ }^{(D)},{ }^{2}$ \\ and Sammy M. Njenga ${ }^{2}$ \\ ${ }^{1}$ College of Health Sciences (COHES), Jomo Kenyatta University of Agriculture and Technology (JKUAT), P. O. Box 62000-00200, \\ Nairobi, Kenya \\ ${ }^{2}$ Eastern and Southern Africa Centre of International Parasite Control (ESACIPAC), Kenya Medical Research Institute (KEMRI), \\ P. O. Box 54840-00200, Nairobi, Kenya
}

Correspondence should be addressed to Elizabeth Njambi; enkimiri@gmail.com

Received 14 September 2019; Revised 20 March 2020; Accepted 7 April 2020; Published 11 May 2020

Academic Editor: Pedro P. Chieffi

Copyright (c) 2020 Elizabeth Njambi et al. This is an open access article distributed under the Creative Commons Attribution License, which permits unrestricted use, distribution, and reproduction in any medium, provided the original work is properly cited.

\begin{abstract}
School children bear a significant burden of intestinal parasitic infections. Because they spend most of their time at home and school, it is necessary to identify the key water, sanitation, and hygiene (WASH) factors associated with these infections in both environments. This was a cross-sectional survey conducted in Mwea West, Kirinyaga County. 180 primary school children aged 8-14 years were randomly selected from three schools (Mianya, Mbui Njeru, and Mukou primary schools). Questionnaires and checklists were administered and single stool samples were collected. Stool samples were microscopically examined for Schistosoma mansoni, soil-transmitted helminths, and protozoan infections. Data on WASH were obtained at home and school. The factors significantly associated with S. mansoni and intestinal protozoa infections in the school children were determined using univariable and multivariable logistic regression models reporting the odds ratio at $95 \%$ confidence intervals. The overall prevalence of S. mansoni and intestinal protozoa infections was 70.5\% (95\% CI: 59.1-84.3) and 32.7\% (95\% CI: 26.8-40.1), respectively. Only one case of STH (A. lumbricoides) was identified. The prevalence of coinfections of S. mansoni and intestinal protozoa infections was $22.8 \%$ (95\% CI: 19.2-27.1). An increased prevalence of S. mansoni infection was associated with children above 12 years $(\mathrm{aOR}=3.19, p=0.015)$, those in Mianya primary $(\mathrm{aOR}=1.23, p=0.001)$, those in Mukou primary $(\mathrm{aOR}=3.19$, $p=0.001)$, and reported behavior of wearing shoes at home $(\mathrm{aOR}=1.67, p=0.010)$. However, handwashing behavior after defecation at home $(\mathrm{aOR}=0.39, p=0.001)$ was protective against $S$. mansoni infection. For any protozoan infection, male children had increased odds of infection $(\mathrm{aOR}=2.41, p=0.001)$ while use of wiping material $(\mathrm{aOR}=0.55, p=0.019)$ and water contact $(\mathrm{aOR}=0.32, p=0.001)$ was protective against intestinal protozoa infections. Infections with $S$. mansoni and any protozoa and their coinfection were present. Findings revealed that several hygiene factors were protective against infections while other were risk factors. Therefore, deworming should be complemented with behavior education on hygienic habits.
\end{abstract}

\section{Introduction}

Intestinal parasitic infections are a major public health concern in developing countries and the risk factors for infection include living in rural areas, poor communities, poor sanitation, lack of clean water, and poor personal hygiene [1]. In addition to these risk factors, low level of awareness resulting in poor hygiene habits leads to schoolaged children suffering the highest infection rates $[2,3]$. These infections are caused by soil-transmitted helminths (STH), Schistosoma mansoni, and intestinal protozoan parasites among others. STH are commonly caused by infection with Ascaris lumbricoides (roundworm), Ancylostoma duodenale, Necator americanus (hookworm), and 
Trichuris trichiura (whipworm) [4]. Schistosomiasis affects almost 240 million people worldwide, and more than 700 million people live in endemic areas where there is presence of water bodies that harbor susceptible species of snails [5].

Information on the prevalence of intestinal protozoa infections is scarce and little data are available from SubSaharan Africa [6]. Studies have shown that Giardia intestinalis and Entamoeba histolytica are the most common pathogenic intestinal protozoa in temperate and tropical countries, especially among children and the elderly, causing severe diarrhea. Transmission of these pathogenic protozoa is by consumption of contaminated water and food [7, 8]. Globally, an estimated 400 million school children are the worst affected by intestinal parasitic infections and due to the similar nature of transmission cycles, coinfections are common [9, 10]. In Sub-Saharan Africa, an estimated 89.9 million school age children are infected, and studies have shown that factors associated with transmission of infections include fecal contamination of water sources, especially the unimproved sources, lack of environmental sanitation, poor socioeconomic conditions, and poor hygiene practices [9-14].

Transmission of these infections among school children can be divided into two routes: home (domestic domain) and school (public domain) where they spend most of their time $[15,16]$. Domestic domain is the geographical location occupied by and under the control of a household while public domain includes public places of work, schooling, commerce, and recreation as well as the streets and fields [16]. Studies have revealed that factors influencing the spread of intestinal parasites were largely centered in the family [16]. In addition, increased risk of transmission has been observed where there was crowding of children (such as schools) due to increased contact and environmental contamination [17].

It has been reported that there is inadequate reliable national data available on the status of school water, sanitation, and hygiene (WASH) in Kenya [18]. In addition, majority of studies performed on WASH and association with infections in school children only captures data from one environment while ignoring other potentially infectious environments [15]. This study determined the prevalence of STH, S. mansoni, and intestinal protozoa infections in school children and investigated the WASH factors associated with their occurrence at home and school.

\section{Materials and Methods}

2.1. Study Area and Population. The study was conducted in Mwea West Sub-County, Kirinyaga County, in Central Kenya. The Mwea irrigation scheme is endemic for $S$. mansoni and STH infections, thus making it an ideal study site $[19,20]$. The county is located about $150 \mathrm{~km}$ northeast of Nairobi. It covers an area of $513 \mathrm{~km}^{2}$; it is estimated to have 51,444 households and a total population of 176,261 people. There are 58,970 school-aged children in Mwea [21]. The main socioeconomic activity in this area is rice farming, which is done through canal irrigation using water from rivers Thiba and Nyamindi. In this study, school-going children aged 8 to 14 years from three primary schools in Mwea West Sub-County were included.

\subsection{Study Design, Sample Size, and Sampling Strategy.} This was a cross-sectional study and a total of 60 children per school were randomly sampled. Sampling was based on the World Health Organization (WHO) guidelines for STH and schistosomiasis surveys and preventive chemotherapy for STH, specifically school-based deworming (SBD) programs $[4,22,23]$. The guideline proposed that, for surveys, a few schools near irrigated areas should be selected and in each school fifty children from the three upper classes should be selected and asked to provide single stool sample. In line with this guideline, three schools in the study area (Mianya, Mukou, and Mbui Njeru primary schools) were purposively selected based on previous studies showing prevalence of $S$. mansoni and STH $[20,24]$. In each school, we randomly sampled 60 children aged 8 to 14 years, who were presumably in classes 2 to 6 . This study included children of this age group because recent studies have showed that intestinal parasites occur mainly in children among this age group [24]. All the selected children provided single stool samples and participated in both the school and household components of the study.

2.3. Field Procedures. The school component of the study was carried out between morning and midmorning hours and the household component was carried out between late afternoon and evening on the same day (15:30 to 18:00 hours). Data collection using piloted questionnaires and checklists was carried out with the help of two trained field assistants familiar with the study area. The field assistants were also responsible for following up the school children to their homes after school.

2.4. School Survey. The research team visited each school prior to the survey and participants were selected using generated random numbers. Parents or guardians of the selected children were invited to a meeting to communicate the study purpose and obtain their consent. All parents/ guardians consented to allow their children to participate in the study and a written informed consent was obtained from them before conducting the study. In addition, assent was sought (verbal) from participating children who were above 13 years. Each participating child was left with a small plastic container (polypot) and instructed to collect a morning stool sample on the day of the survey, and the plastic containers containing morning stool samples were collected from each child. Upon receipt of the stool sample, each of the stool container was labelled with the participant's unique identification number. The samples were kept in a cooler box and transported to Kimbimbi Sub-County hospital laboratory for microscopic screening within the same day. Thereafter, each child responded to an interviewer-administered questionnaire on WASH which had been pretested at a local school in Mwea and was adapted before administration. 
2.5. Household Survey. Using a checklist, direct observations were carried out at each participating child's home to investigate the water sources and sanitation facilities. This was performed with the assistance of the head of household or any adult family member (above 18 years old).

2.6. Data Collection Tools. A questionnaire was developed to obtain information on children's demographic data, hygiene habits, eating and playing habits, handwashing behavior at critical times, and use of water and sanitation facilities. Direct observations on water sources and sanitation facilities were carried out at the school compound and at the homes of the children. Water sources and sanitation facilities were defined using the Joint Monitoring Programme (JMP) guidelines of the WHO and the United Nations Children Fund (UNICEF) for water and sanitation [25]. Improved sanitation facilities were defined as availability of flush toilet, toilet connected to a piped sewer system, toilet connected to a septic system, flush to a pit latrine, ventilated improved pit latrine (VIP), and pit latrine with slab and composting toilet. Improved water sources were defined as availability of piped water into dwelling, piped water into yard/plot, public tap or stand pipe, borehole, protected well or spring, bottled water, and rain water. The condition of the toilets (cleanliness) was also observed as well as presence of handwashing facilities and anal cleansing material in the toilets.

2.7. Laboratory Procedures. The specimens were checked for identification number, quantity, and quality (no urine or dirt) and divided into two aliquots. One aliquot was used for microscopic screening of $S$. mansoni and STH infections using double slide Kato-Katz technique while the other was used to screen for protozoan infection using direct saline and iodine wet mount methods. Quality of reagents and instruments was checked by laboratory technicians.

2.8. Helminths Screening. Each stool specimen was analyzed for S. mansoni and STH infections using the double slide Kato-Katz thick smears method [26]. Briefly, the stool sample was passed through a metal sieve to remove fibrous material. Using a spatula, some amount of stool was collected and filled in a template on a slide. Cellophane soaked in glycerine malachite green was placed on the smear and the slide was turned upside down, pressed, and allowed to spread evenly. After a clearance time of 30 minutes, the slide was examined under a light microscope (100x magnification). Egg counts per slide was multiplied by a factor of 24 to obtain eggs per gram of feces (epg) [22].

2.9. Protozoa Screening. For each sample, two wet preparations were made for saline wet mount and iodine wet mount tests $[27,28]$. For each of the preparations, approximately 2 grams of stool sample was picked up using a wooden stick and placed on two separate glass slides. The first preparation was mixed with a drop of normal saline $(0.9 \%)$ and the second with a drop of dilute Lugol's iodine and normal saline (1:5 distilled water). Both slides were covered with a cover slip and observed under the microscope at $10 \mathrm{x}$ and at $40 \mathrm{x}$ magnifications. Saline preparation and iodine allowed for visualization of motile trophozoites and cysts, respectively.

2.10. Data Management and Statistical Analysis. Data collected were checked to ensure accuracy and completeness on site before double entry into a Microsoft Excel spreadsheet. The observed prevalence of $S$. mansoni and intestinal protozoa infections were calculated by school, as well as by demographic variables, and the $95 \%$ confidence intervals (CIs) were obtained using binomial logistic regression, taking into account clustering by schools. For purposes of this analysis, the following age groups were used: $<10,10-12$, and $>12$ years. Using univariable analysis, factors associated with $S$. mansoni and intestinal protozoa infections were determined and described as odds ratio (OR). Minimum adequate variables for multivariable analysis were selected by specifying an inclusion criterion of $p$ value $<0.05$ in a sequential (block-wise) variable selection method. Adjusted odds ratios (aOR) were obtained by mutually adjusting all minimum generated variables using a multivariable logistic regression model. All statistical analyses were carried out using STATA version 14.1 (STATA Corporation, College Station, TX, USA).

\section{Results}

3.1. Sociodemographic, Household, and School Characteristics. The overall data were collected from 180 children in three primary schools in Mwea West. The mean age of children was 10.0 years (range: $7-15$ years, standard deviation $(\mathrm{SD})=$ 1.6 years). There was an equal representation of study participants in terms of gender $(50.0 \% / 50.0 \%)$ females/ males. Majority of the study participants were in the age group 10-12 years (99 participants; $55 \%$ ), followed by those below 10 years (66 participants; 36.6\%), and those above 12 years (15 participants; $8.3 \%$ ).

Out of all the households surveyed, 35.6\% (64 households) used improved water sources and 152 (84\%) used improved latrines (VIP). All three schools used improved sources of water for drinking and had improved latrine facilities (VIP). For personal and environmental hygiene purposes such as handwashing and cleaning classrooms, Mianya and Mbui Njeru used water from unimproved sources (surface water) while Mukou used piped water (improved sources). The pupil per latrine ratio was $23: 1,67$ : 1, and 17:1 for Mianya, Mbui Njeru, and Mukou primary schools, respectively.

3.2. Prevalence of S. mansoni and STH Infections. Only one case of STH (A. lumbricoides) was identified. The overall prevalence of S. mansoni was 70.5\% (95\% CI: 59.0\%-84.2\%). The mean infection intensity was 376.2 epg (95\% CI: 222.3-530.1) and was categorized in overall as moderate infection. Majority of the infections were light infections 46 (25.6\%), followed by moderate infections 41 (22.8\%) and severe infections $40(22.2 \%)$. Out of the three schools, 
Mukou primary had the highest prevalence of infection, 83.3\% (95\% CI: 74.4.4-93.3, $p=0.002$ ), followed by Mianya primary, 65.0\% (95\% CI: 53.9-78.3, $p=0.001)$ and Mbui Njeru primary, 63.3\% (95\% CI: $52.2-76.8, \quad p=0.001$ ) (Figure 1). Children aged above 12 years had a higher prevalence of $86.6 \%$ (95\% CI: 81.4-98.3, $p=0.001$ ), followed by those aged 10 to 12 years, $71.7 \%$ (95\% CI: $54.5-94.4$, $p=0.018$ ), and those below 10 years, $65.2 \%$ (95\% CI: 49.4-85.9, $p=0.002$ ). The male gender reported a higher prevalence of infection compared to the female gender, 73.3\% (95\% CI: 62.9-82.1, $p=0.001$ ) and 67.7\% (95\% CI: $57.0-77.2, p=0.002$ ), respectively (Table 1 ).

3.3. Prevalence of Intestinal Protozoa Infection. The overall prevalence of protozoan infections was 32.7\% (95\% CI: 26.7-40.1). Out of the three schools, Mianya primary had a higher prevalence of infection, 38.3\% (95\% CI: 27.8-52.8, $p=0.001$ ), followed by Mbui Njeru, 33.3\% (95\% CI: 23.3-47.7, $p=0.001$ ), and Mukou primary, 26.7\% (95\% CI: $17.5-40.6, p=0.0001$ ) (Figure 1). A slightly higher prevalence was reported in females compared to males, $33.3 \%$ (95\% CI: 23.7-44.0, $p=0.001)$ and $32.2 \%$ (95\% CI: 22.7-42.9, $p=0.001$ ), respectively. Children aged between 10 to 12 years had the highest prevalence, $34.3 \%$ (95\% CI: 22.3-52.9, $p=0.001$ ), followed by those above 12 years, $33.3 \%$ (95\% CI: 21.2-52.4, $p=0.003$ ), and those below 10 years, 30.3\% (95\% CI: 19.3-47.6, $p=0.001)$. The most prevalent species was Entamoeba coli infections, 18.8\% (95\% CI: $17.8-20.0, p=0.001$ ), followed by $E$. histolytica/dispar infections, $12.2 \%$ (95\% CI: 6.5-22.8, $p=0.001$ ), and $G$. intestinalis infection, $8.3 \%$ (95\% CI: 3.8-18.2, $p=0.001$ ) (Table 1).

3.4. Prevalence of Coinfections. The overall prevalence of $S$. mansoni and any protozoa infection was $22.8 \%$ (95\% CI: 19.2-27.1). Prevalence in Mianya primary was the highest, 26.7\% (95\% CI: $17.5-40.6, p=0.001$ ), then Mukou primary, 21.7\% (95\% CI: 13.4-35.1, $p=0.001$ ), and Mbui Njeru primary, $20.0 \%$ (95\% CI: 12.1-33.2, $p=0.001$ ). Coinfections were highest among those aged above 12 years, 33.3\% (95\% CI: $21.2-52.4, p=0.003)$, followed by those aged below 10 years, $24.2 \%$ (95\% CI: $14.0-41.9, p=0.001$ ), and those between 10-12 years, 20.2\% (95\% CI: 16.1-25.3, $p=0.001$ ). Also, female children had a greater prevalence compared to males, 25.6\% (95\% CI: 16.3-40.1, $p=0.001)$ and 20.0\% (95\% CI: 16.6-24.2, $p=0.001)$, respectively.

3.5. Univariable Analysis of Risk Factors. Factors associated with greater odds of $S$. mansoni infection were pupils at Mukou primary school $(\mathrm{OR}=2.69, p=0.024)$, children aged above 12 years $(\mathrm{OR}=3.48, p=0.002)$, and handwashing after defecation at school $(\mathrm{OR}=1.22, p=0.001)$. Handwashing after defecation at home $(\mathrm{OR}=0.48$, $p=0.001)$ was protective for $S$. mansoni infection. Pupils at Mbui Njeru and Mukou primary $(\mathrm{OR}=0.80, p=0.001)$ $(\mathrm{OR}=0.58, p=0.001)$, respectively had lower odds of protozoa infections. Also, use of wiping material $(\mathrm{OR}=0.64$,

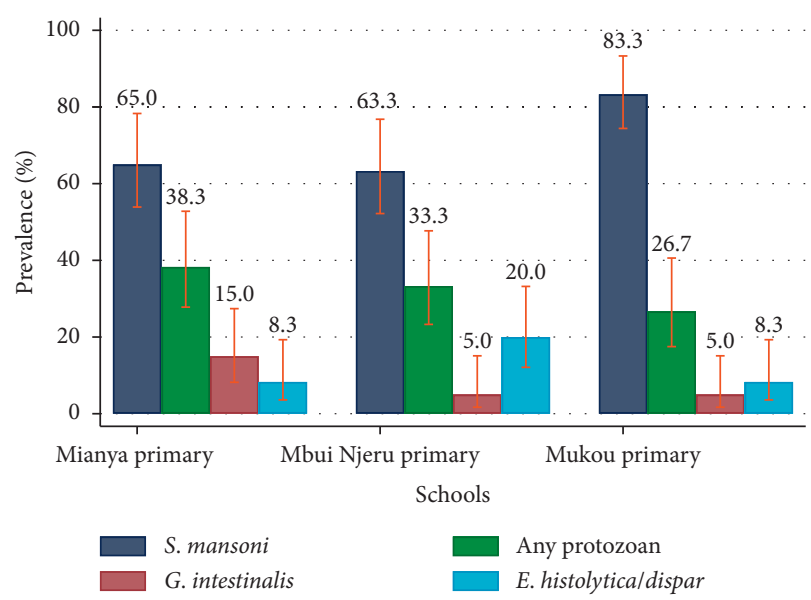

FIgURE 1: Prevalence of intestinal parasitic infections in school children per school.

$p=0.001)$, contact with water bodies $(\mathrm{OR}=0.70$, $p=0.001)$, and presence of a toilet handwashing set-up $(\mathrm{OR}=0.78, p=0.034)$ while adequately cleaned toilets at home slightly increased the risk of protozoan infections $(\mathrm{OR}=1.84, p=0.040)($ Table 2$)$.

3.6. Multivariable Analysis of Risk Factors. Factors-associated increased prevalence of $S$. mansoni infection was children above 12 years $(\mathrm{aOR}=3.19, p=0.015)$, Mianya primary $(\mathrm{aOR}=1.23, p=0.001)$, Mukou primary $(\mathrm{aOR}=3.19$, $p=0.001$ ), and reported behavior of wearing shoes at home $(\mathrm{aOR}=1.67, p=0.010)$. Handwashing behavior after defecation at home $(\mathrm{aOR}=0.39, p=0.001)$ was protective against $S$. mansoni infection. For any protozoan infection, male children had increased odds of infection $(\mathrm{aOR}=2.41, p=0.001)$ while use of wiping material $(\mathrm{aOR}=0.55, p=0.019)$ and water contact $(\mathrm{aOR}=0.32$, $p=0.001)$ was protective against intestinal protozoa infections (Table 3).

Variables selected for S. mansoni infection: school, age group, gender, handwashing after defecation at home, type of wiping material used, and handwashing after defecation at school.

Variables selected for any protozoan infection: school, gender, use of wiping material, contact with water bodies, presence of a toilet handwashing station, and clean latrines.

\section{Discussion}

In Kenya, schistosomiasis caused by $S$. mansoni occurs mostly in the central and western parts of the country $[29,30]$, and in cases where S. mansoni infections are a public health concern, the WHO recommends for mass drug administration (MDA) [22]. In this study, overall prevalence of S. mansoni was higher compared to findings from other past studies conducted in the study area. In 2015, prevalence of S. mansoni infection was $53.7 \%$ while in 2012 it was $47.4 \%$ $[24,31]$, respectively. This shows that reinfection is on the rise after withdrawal of MDA. Therefore, the study population requires consistent annual treatment campaigns 
TABle 1: Prevalence of S. mansoni and intestinal protozoa infections by categories, age, sex, and school.

\begin{tabular}{|c|c|c|c|c|c|}
\hline & $\begin{array}{l}\text { S. mansoni } \\
n / N(\%)\end{array}$ & $\begin{array}{c}\text { Any intestinal protozoan } \\
n / N(\%)\end{array}$ & $\begin{array}{l}\text { G. intestinalis } \\
n / N(\%)\end{array}$ & $\begin{array}{c}\text { E. histolytica/dispar } \\
n / N(\%)\end{array}$ & $\begin{array}{c}\text { E. coli } \\
n / N(\%)\end{array}$ \\
\hline Overall prevalence & $127(70.5 \%)$ & $59(32.7 \%)$ & $15(8.3 \%)$ & $22(12.2 \%)$ & $34(18.9 \%)$ \\
\hline \multicolumn{6}{|l|}{ Prevalence by age } \\
\hline$<10$ years & $65.10 \%$ & $30.30 \%$ & $5(7.6 \%)$ & $7(10.6 \%)$ & $12(18.2 \%)$ \\
\hline $10-12$ years & $71.70 \%$ & $34.30 \%$ & $10(10.1 \%)$ & $14(14.1 \%)$ & $19(19.2 \%)$ \\
\hline$>12$ years & $86.60 \%$ & $33.30 \%$ & 0 & $1(6.7 \%)$ & $3(20.0 \%)$ \\
\hline \multicolumn{6}{|c|}{ Prevalence by gender } \\
\hline Male & $73.30 \%$ & $32.20 \%$ & $7(7.8 \%)$ & $9(10.0 \%)$ & $16(17.8 \%)$ \\
\hline Female & $67.70 \%$ & $33.30 \%$ & $8(8.9 \%)$ & $13(14.4 \%)$ & $18(20.0 \%)$ \\
\hline \multicolumn{6}{|l|}{ Prevalence by school } \\
\hline Mianya & $65.00 \%$ & $38.30 \%$ & $9(15.0 \%)$ & $5(8.3 \%)$ & $11(18.3 \%)$ \\
\hline Mbui Njeru & $63.30 \%$ & $33.30 \%$ & $3(5.0 \%)$ & $12(20.0 \%)$ & $11(18.3 \%)$ \\
\hline Mukou & $83.30 \%$ & $26.60 \%$ & $3(5.0 \%)$ & $5(8.3 \%)$ & $12(20.0 \%)$ \\
\hline
\end{tabular}

[22]. In the Mwea irrigation scheme, high prevalence of $S$. mansoni can be attributed to rich farming which leads to repeated contact with water bodies where snails, the intermediate hosts of schistosomiasis, breed [32]. Further, due to lack of sanitation facilities in the farms, the water in irrigation canals is contaminated with feces and therefore contact with the water leads to transfer of infections [33].

A significantly higher prevalence of $S$. mansoni was observed in children above 12 years and these findings can be explained by the fact that children in this age group are more involved in household chores, farming, or recreational activities potentially bringing them in contact with water [34]. Similar findings were observed in a study in Western Kenya where prevalence peaked in early adolescence $(p<0.003)$ [35]. This present study did not reveal gender as a risk factor for $S$. mansoni infection. However, male school children had slightly greater odds of infection as compared to female children. This may be due to the playful habits of boys compared to girls which may put them in contact with infected water. This corroborates findings of a study conducted in the same area which reported a higher prevalence in males than females [24].

As has been mentioned earlier, information on the prevalence of intestinal protozoa infections is scarce and little data are available from Sub-Saharan Africa [6]. Therefore, determining the prevalence of these infections in school children was a key objective. The observed prevalence of intestinal protozoa was similar to findings in a study conducted in Thika town, Central Kenya, 32.7\% and 38.9\%, respectively [36]. Similarly, in the Thika study, the presence of infections from pathogenic species was also identified, specifically Entamoeba histolyticaldispar (14.6\%) and Giardia lamblia (6.9\%). Findings from a study in Murang'a County in Central Kenya also observed infections with intestinal protozoa among school-aged children [37]. This shows that infections with intestinal protozoa infections are overlooked and should be considered in future survey and MDA activities.

Coinfections with S. mansoni and any intestinal protozoa infection were also observed among the primary school children. Multiple infections are common probably because of similarities in modes of transmission. Among primary school children in Murang'a County, prevalence of coinfection was $9.3 \%$ and the most frequent combination was between E. histolytica and A. lumbricoides [37]. Similarly, multiple infections in Ethiopian school children were $6.2 \%$ and S. mansoni and intestinal protozoa coinfection was observed although to a smaller degree [38]. Infections with intestinal helminths are a common occurrence among primary school children in Kenya with ascariasis, trichuriasis, and hookworm infections as the most common [31, 36, 39]. However, in this study, only one case of ascariasis was detected. This may be because the Kato Katz technique used for screening of STH infection was not a sensitive method and may have missed light infections.

In order to reduce reinfection, there is need to focus on WASH factors which have been associated with intestinal parasitic infections. These infections may occur at home or at school because children spend most of their time in these environments $[15,16]$. This study investigated WASH factors associated with infections at homes and schools. In the study, the behavior of handwashing after defecation at home was protective against $S$. mansoni infections. This agrees with studies that have shown hygienic habits, such as handwashing, which play a significant role in preventing transmission of these infections [40]. However, wearing shoes in school children at home was associated with increased risk of $S$. mansoni infections. This may be because children did not consistently wear shoes, therefore increasing exposure to infected snails.

The study found that male children had increased odds of intestinal protozoa infections than the females. A study in Ethiopian primary school children observed the same association [38]. This higher prevalence may be attributed to the adventurous nature of male children which increases their risk of infections when they are outdoors as compared to females. Also, the use of wiping material was found to be protective against intestinal protozoa and this reaffirms that hygienic behaviors are significant in preventing transmission. Water contact was also seen as protective against intestinal protozoa. The transmission of intestinal protozoa infections is via fecal-oral route and therefore contact water would not be a risk for these infections [41]. Studies have shown that open defecation has been significantly associated with intestinal parasitic infections (including E. histolytica/dispar) [42, 43]. A study in West Africa revealed that E. histolytica/dispar had a 
TABLe 2: Univariable analysis of risk factors of S. mansoni and intestinal protozoa infections.

\begin{tabular}{|c|c|c|c|c|c|c|}
\hline \multirow[b]{2}{*}{ Characteristic } & \multirow[b]{2}{*}{$\begin{array}{l}N=180, n \\
\quad(\%)\end{array}$} & \multicolumn{5}{|c|}{ Univariable analysis [OR, $p$-value] } \\
\hline & & $\begin{array}{l}\text { S. mansoni } \\
(n=127)\end{array}$ & $\begin{array}{l}\text { Any protozoan } \\
\quad(n=59)\end{array}$ & $\begin{array}{l}\text { G. intestinalis } \\
(n=15)\end{array}$ & $\begin{array}{l}\text { E. histolytical } \\
\text { dispar }(n=22)\end{array}$ & E. $\operatorname{coli}(n=34)$ \\
\hline \multicolumn{7}{|l|}{$\begin{array}{l}\text { Demographics } \\
\text { School }\end{array}$} \\
\hline Mianya & $60(33.3)$ & Reference & & & & \\
\hline Mbui Njeru & $60(33.3)$ & $0.94, p=0.849$ & $0.80, p=0.001^{*}$ & $0.29, p=0.081$ & $2.75, p=0.075$ & $1.00, p=1.000$ \\
\hline Mukou & $60(33.3)$ & $2.69, p=0.024^{*}$ & $0.58, p=0.001^{*}$ & $0.29, p=0.081$ & $1.00, p=1.000$ & $1.11, p=0.817$ \\
\hline \multicolumn{7}{|l|}{ Age group } \\
\hline$<10$ years & $66(36.7)$ & Reference & & & & \\
\hline $10-12$ years & $99(55.0)$ & $1.36, p=0.634$ & $1.20, p=0.746$ & $1.37, p=0.737$ & $1.39, p=0.591$ & $1.07, p=0.949$ \\
\hline$>12$ years & $15(8.3)$ & $3.48, p=0.002^{*}$ & $1.15, p=0.832$ & - & $0.60, p=0.604$ & $1.13, p=0.456$ \\
\hline \multicolumn{7}{|l|}{ Gender } \\
\hline Female & $(50.0)$ & Reference & & & & \\
\hline Male & $90(50.0)$ & $1.31, p=0.429$ & $0.95, p=0.817$ & $0.86, p=0.884$ & $0.66, p=0.001^{*}$ & $0.86, p=0.056$ \\
\hline \multicolumn{7}{|l|}{$\begin{array}{l}\text { Individual and household } \\
\text { characteristics }\end{array}$} \\
\hline Open defecation behavior & $90(50.8)$ & $1.17, p=0.410$ & $0.66, p=0.227$ & $0.62, p=0.001^{*}$ & $0.34, p=0.039^{*}$ & $0.60, p=0.036^{*}$ \\
\hline Use of wiping material & $63(70.8)$ & $1.56, p=0.550$ & $0.64, p=0.001^{*}$ & $2.16, p=0.389$ & $0.18, p=0.001^{*}$ & $0.61, p=0.011^{*}$ \\
\hline Contact with water bodies & $160(88.9)$ & $0.78, p=0.659$ & $0.70, p=0.001^{*}$ & $1.82, p=0.493$ & $0.76, p=0.212$ & $0.49, p=0.161$ \\
\hline $\begin{array}{l}\text { Eating unwashed food/ } \\
\text { fruits }\end{array}$ & $46(25.6)$ & $1.25, p=0.784$ & $1.12, p=0.673$ & $0.71, p=0.020^{*}$ & $1.11, p=0.847$ & $0.87, p=0.796$ \\
\hline $\begin{array}{l}\text { Handwashing after } \\
\text { defecation at home }\end{array}$ & $163(90.6)$ & $0.48, p=0.001^{*}$ & $0.51, p=0.349$ & $0.37, p=0.141$ & $0.40, p=0.144$ & $1.09, p=0.950$ \\
\hline $\begin{array}{l}\text { Handwashing before } \\
\text { eating at home }\end{array}$ & $161(89.4)$ & $1.12, p=0.234$ & $0.82, p=0.178$ & $0.43, p=0.250$ & $0.71, p=0.628$ & $1.27, p=0.629$ \\
\hline $\begin{array}{l}\text { Behavior of wearing shoes } \\
\text { at home }\end{array}$ & $162(90.5)$ & $1.77, p=0.036$ & $0.89, p=0.745$ & $0.65, p=0.063$ & $2.38, p=0.058$ & $0.74, p=0.543$ \\
\hline $\begin{array}{l}\text { Presence of wiping } \\
\text { material in the latrine }\end{array}$ & $57(31.7)$ & $0.86, p=0.543$ & $0.92, p=0.808$ & $1.49, p=0.179$ & $0.59, p=0.198$ & $1.04, p=0.932$ \\
\hline \multicolumn{7}{|l|}{ Type of wiping material } \\
\hline Toilet paper & $26(45.6)$ & Reference & & & & \\
\hline Leaves & $2(3.5)$ & $0.37, p=0.002^{*}$ & - & - & - & - \\
\hline Newspaper & $29(50.9)$ & $0.70, p=0.050$ & $0.85, p=0.708$ & $1.92, p=0.270$ & $0.57, p=0.001^{*}$ & $0.43, p=0.132$ \\
\hline $\begin{array}{l}\text { Presence of a toilet } \\
\text { handwashing station }\end{array}$ & $163(90.6)$ & $1.42, p=0.256$ & $0.78, p=0.034^{*}$ & $0.41, p=0.161$ & $1.62, p=0.095$ & $0.85, p=0.376$ \\
\hline Damaged toilet structure & $32(17.8)$ & $1.61, p=0.418$ & $0.63, p=0.412$ & $1.17, p=0$. & $0.70, p=0.497$ & $0.56, p=0.248$ \\
\hline Clean latrines & $137(76.1 \%)$ & $1.21, p=0.672$ & $1.84, p=0.040^{*}$ & - & $1.47, p=0.388$ & $1.26, p=0.155$ \\
\hline Improved sources of water & $65(36.1)$ & $1.45, p=0.265$ & $1.08, p=0.584$ & $1.19, p=0.619 p$ & $1.56, p=0.203$ & $1.12, p=0.537$ \\
\hline $\begin{array}{l}\text { Taken deworming } \\
\text { medication }\end{array}$ & $133(73.9)$ & $1.74, p=0.094$ & $0.81, \mathrm{p}=0.745$ & $1.45, p=0.692$ & $0.46, p=0.056$ & $0.68, p=0.243$ \\
\hline $\begin{array}{l}\text { Handwashing after } \\
\text { defecation at school }\end{array}$ & $155(86.6)$ & $1.22, p=0.001^{*}$ & $0.98, p=0.988$ & $1.00, p=0.984$ & $0.47, p=0.571$ & $1.20, p=0.907$ \\
\hline $\begin{array}{l}\text { Handwashing before } \\
\text { eating at school }\end{array}$ & $159(88.3)$ & $0.95, p=0.870$ & $0.97, p=0.960$ & $0.85, p=0.433$ & $0.81, p=0.722$ & 2.39, $p=0.574$ \\
\hline $\begin{array}{l}\text { Behavior of wearing shoes } \\
\text { at school }\end{array}$ & $158(88.3)$ & $0.94, p=0.876$ & $0.32, p=0.113$ & $0.85, p=0.843$ & $0.82, p=0.625$ & $0.32, p=0.039^{*}$ \\
\hline
\end{tabular}

*Significant association $(p<0.05)$; - variable omitted because of insufficient observations.

prevalence of $14.4 \%$ and open defecation was common [43]. Similar findings were observed in the current study although there was no significant association.

Pupil-to-latrine ratio is used to determine whether a school's sanitation facilities are adequate. This has been associated with increased toilet use which leads to better sanitation conditions interrupting transmission of infections [44]. According to the Kenyan government, the recommended pupil-to-latrine ratio is 25:1 and 30:1 for girls and boys, respectively [45]. Out of the three schools, Mianya and Mukou primary schools met the required pupil-to-latrine ratio for both girls and boys. Mbui Njeru primary did not meet the recommendation and school children may have an increased risk of infection. Therefore, construction of additional latrines is necessary in order to meet the government's recommended ratio [46].

\section{Limitations}

Information on hygiene behavior in school children was selfreported which may be subjective and unreliable compared to observing these behaviors. The WASH factors at school were 
TABLE 3: Multivariable analysis of risk factors of S. mansoni and any intestinal protozoa infection.

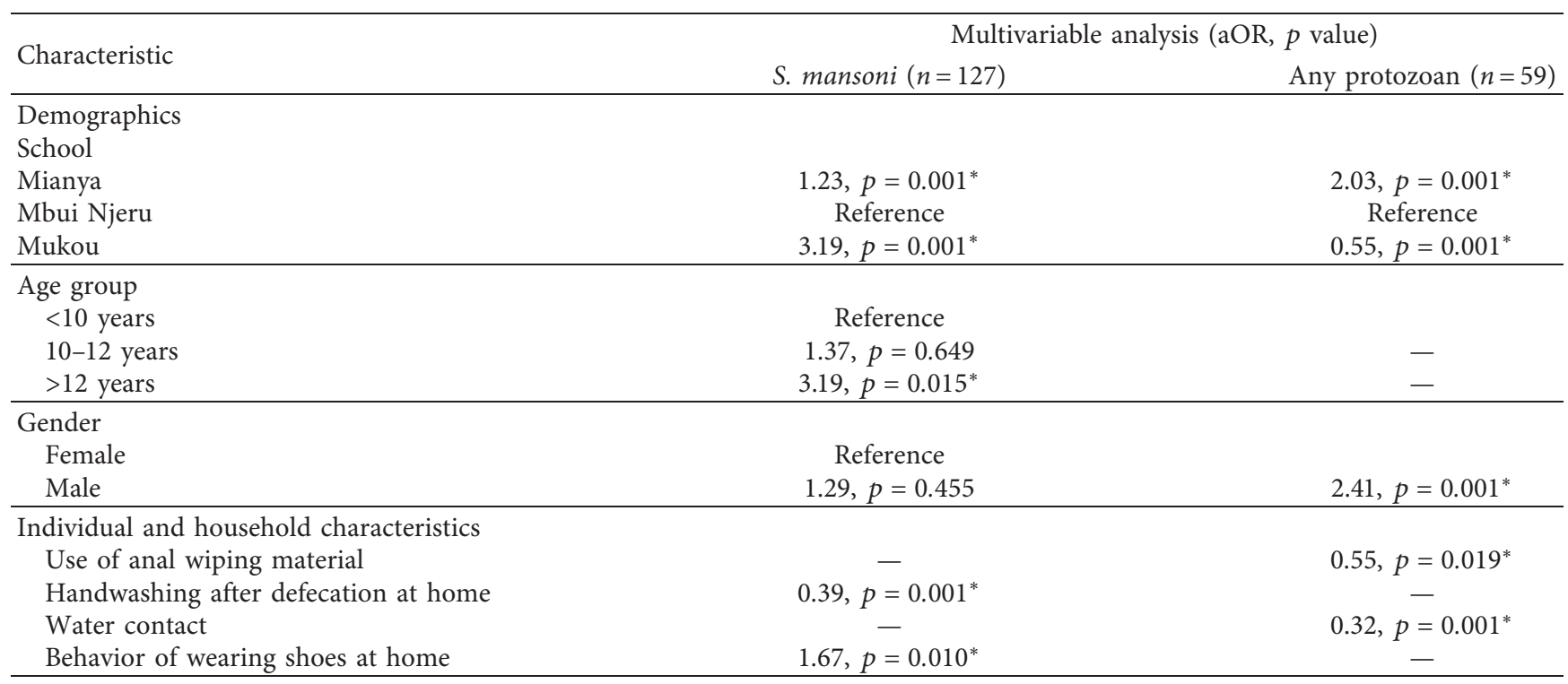

* Significant association $(p<0.05)$.

only collected from three schools and may therefore be inadequate to make inferences. The laboratory methods used for the detection of the intestinal parasitic infections were of limited sensitivity and may have underestimated the prevalence of infections. Identification of the pathogenic E. histolytica from the enteric commensals $E$. dispar was not differentiated due to lack of laboratory facilities in the department.

\section{Conclusion}

Infections with S. mansoni and intestinal protozoa as well as their coinfection are a public health problem in school children in the Mwea irrigation scheme. The hygiene behavior of handwashing at home was protective against $S$. mansoni infections while use of wiping material and water contact was protective against any protozoan infection. However, wearing shoes at home was a risk factor for $S$. mansoni infection. These findings show that WASH factors at the household level have a significant role in transmission. Also, consistency of deworming for S. mansoni infections and treatment for intestinal protozoa infections are necessary. These treatments should be complemented with improvements in school and household WASH conditions and behavior education on hygienic habits.

\section{Abbreviations}

aOR: $\quad$ Adjusted odds ratio

BCC: Behavior change communication

CI: $\quad$ Confidence interval

epg: $\quad$ Eggs per gram of feces

ESACIPAC: Eastern and Southern Africa Centre of International Parasite Control

KEMRI: Kenya Medical Research Institute

MDA: $\quad$ Mass drug administration

OR: $\quad$ Odds ratio

SBD: $\quad$ School-based deworming
SD: $\quad$ Standard deviation

SERU: $\quad$ Scientific Ethics and Review Unit

STH: $\quad$ Soil-transmitted helminths

WASH: Water, sanitation, and hygiene

WHO: World Health Organization.

\section{Data Availability}

The datasets used to support the findings of this study are available from the corresponding author upon request.

\section{Ethical Approval}

The study protocol received ethical approval from the Kenya Medical Research Institute (KEMRI), Scientific Ethics and Review Unit (SERU no. 3093). Permission to conduct the study was sought from the County Health and Education Authorities. School authorities and parents/guardians were informed about the study, and participation was fully voluntary.

\section{Consent}

Written informed consent and verbal assent were obtained from participants' guardian/parent and children above 13 years of age, respectively. Treatment was administered to participants infected with intestinal parasitic infections by a medical professional. For S. mansoni infections, $40 \mathrm{mg} / \mathrm{kg}$ body weight of praziquantel was administered according to the WHO guidelines [22]. Intestinal protozoa infections were treated with metronidazole $20 \mathrm{mg} / \mathrm{kg}$ per day for 5 days [12].

\section{Conflicts of Interest}

The authors declare that there are no conflicts of interest regarding the publication of this article. 


\section{Authors' Contributions}

Elizabeth Njambi participated in the survey design, data collection, and manuscript development. Collins Okoyo participated in the data analysis and manuscript development. Janet Masaku was responsible for fieldwork supervision. Dennis Magu participated in drafting the manuscript. Sammy Njenga was responsible for the study design and overall scientific guidance. All authors read and approved the final paper.

\section{Acknowledgments}

This was a self-funded project. Resources were partly contributed by Prof. Sammy Njenga who has been involved in guiding the overall project. The authors are grateful for the support of the County Health and Education Departments of Kirinyaga County, the teachers, parents, and children who participated in the study. Mr. Lawrence Muthami is thanked for guidance in preparing an earlier draft manuscript of this paper. Also, special thanks to the Eastern and Southern Africa Centre of International Parasite Control (ESACIPAC), Kenya Medical Research Institute (KEMRI), and Kimbimbi Sub-County Hospital for laboratory and logistical support.

\section{References}

[1] V. Mehraj, J. Hatcher, S. Akhtar, G. Rafique, and M. A. Beg, "Prevalence and factors associated with intestinal parasitic infection among children in an urban slum of Karachi," PLoS One, vol. 3, no. 11, Article ID e3680, 2008.

[2] T. Alelign, A. Degarege, and B. Erko, "Soil-transmitted helminth infections and associated risk factors among schoolchildren in Durbete town, northwestern Ethiopia," Journal of Parasitology Research, vol. 2015, Article ID 641602, 5 pages, 2015.

[3] P. Okyay, S. Ertug, B. Gultekin, O. Onen, and E. Beser, "Intestinal parasites prevalence and related factors in school children, a western city sample-Turkey," BMC Public Health, vol. 4, p. 64, 2004.

[4] WHO, "preventive chemotherapy in human helminthiasis use anthelminthic drugs control," WHO, Geneva, Switzerland, 2006, http://whqlibdoc.who.int/publications/2006/ 9241547103_eng.pdf\%5Cnhttp://scholar.google.com/ scholar?hl=en\&btnG=Search\&q=intitle:

Preventive+chemotherapy+in+human+helminthiasis\# 8.

[5] World Health Organization, "WHO | What is schistosomiasis ?” World Health Organisation, Geneva, Switzerland, 2016, https://www.who.int/schistosomiasis/disease/en/.

[6] B. Speich, H. Marti, S. M. Ame et al., "Prevalence of intestinal protozoa infection among school-aged children on Pemba Island, Tanzania, and effect of single-dose albendazole, nitazoxanide and albendazole-nitazoxanide," Parasites \& Vectors, vol. 6, no. 1, p. 3, 2013.

[7] Y. Mohammed, M. Aliyu, N. Dabo, N. Adabara, B. Otone, and A. Ige, "Prevalence of intestinal Protozoan parasites infection among primary school pupils in bosso local government area, Niger state, Nigeria," African Journal of Clinical and Experimental Microbiology, vol. 16, p. 45, 2015.

[8] B. Berhe, G. Bugssa, S. Bayisa, and M. Alemu, "Foodborne intestinal protozoan infection and associated factors among patients with watery diarrhea in Northern Ethiopia; a crosssectional study," Journal of Health, Population and Nutrition, vol. 37, no. 1, p. 5, 2018.

[9] C.-W. Liao, C.-J. Fu, C.-Y. Kao et al., "Prevalence of intestinal parasitic infections among school children in capital areas of the Democratic Republic of São Tomé and Príncipe, West Africa," African Health Sciences, vol. 16, no. 3, pp. 690-697, 2016.

[10] A. A. Obala, C. J. Simiyu, D. O. Odhiambo et al., "Webuye health and demographic surveillance systems baseline survey of soil-transmitted helminths and intestinal Protozoa among children up to five years," Joyrnal of Tropical Medicine, vol. 2013, Article ID 734562, 7 pages, 2013.

[11] X. Villamizar, A. Higuera, G. Herrera et al., "Molecular and descriptive epidemiology of intestinal protozoan parasites of children and their pets in Cauca, Colombia: a cross-sectional study," BMC Infectious Diseases, vol. 19, no. 1, pp. 1-11, 2019.

[12] B. Matthys, M. Bobieva, G. Karimova et al., "Prevalence and risk factors of helminths and intestinal protozoa infections among children from primary schools in western Tajikistan," Parasites and Vectors, vol. 4, no. 1, p. 195, 2011.

[13] S. Erismann, S. Diagbouga, P. Odermatt et al., "Prevalence of intestinal parasitic infections and associated risk factors among schoolchildren in the plateau central and centre-ouest regions of burkina faso," Parasites \& Vectors, vol. 9, p. 554, 2016.

[14] S. Brooker, A. C. A. Clements, and D. A. P. Bundy, "Global epidemiology, ecology and control of soil-transmitted helminth infections," Advances in Parasitology, vol. 62, pp. 221-261, 2006.

[15] M. C. Freeman, A. N. Chard, B. Nikolay et al., "Associations between school- and household-level water, sanitation and hygiene conditions and soil-transmitted helminth infection among Kenyan school children," Parasites \& Vectors, vol. 8, p. $412,2015$.

[16] S. Cairncross, U. Blumenthal, P. Kolsky, L. Moraes, and A. Tayeh, "The public and domestic domains in the transmission of disease," Tropical Medicine \& International Health, vol. 1, no. 1, pp. 27-34, 1996.

[17] C. K. Mbae, D. J. Nokes, E. Mulinge, J. Nyambura, A. Waruru, and S. Kariuki, "Intestinal parasitic infections in children presenting with diarrhoea in outpatient and inpatient settings in an informal settlement of Nairobi, Kenya," BMC Infectious Diseases, vol. 13, p. 243, 2013.

[18] UNICEF, WASH in Schools, 2014.

[19] B. A. Okech, I. K. Mwobobia, A. Kamau et al., "Use of integrated malaria management reduces malaria in kenya," PLoS One, vol. 3, p. 12, 2008.

[20] J. H. Kihara, N. Muhoho, D. Njomo et al., "Drug efficacy of praziquantel and albendazole in school children in mwea division, central province, kenya," Acta Tropica, vol. 102, no. 3, pp. 165-171, 2007.

[21] Kenya National Bureau of Statistics (KNBS), The 2009 Kenya Population and Housing Census-Population Distribution by Age, Sex and Administrative Units, Kenya National Bureau Statistics, Nairobi, Kenya, 2010.

[22] A. Montresor, D. Crompton, A. Hall, DA. Bundy, and L. Savioli, Guidelines for the Evaluation of Soil-Transmitted Helminthiais and Schistomiasis at Community Level, WHO, Geneva, Switzerland, 1998.

[23] Deworm the World, School-Based Deworming: A Planner's Guide to Proposal Development for National School-Based Deworming Programs, Deworm the World, Washington, DC, USA, 2010, http://www.who.int/wormcontrol/en/. 
[24] J. Masaku, N. Madigu, C. Okoyo, and S. M. Njenga, "Current status of Schistosoma mansoni and the factors associated with infection two years following mass drug administration programme among primary school children in Mwea irrigation scheme: a cross-sectional study," BMC Public Health, vol. 15, p. 739, 2015.

[25] World Health Organization, WHO/UNICEF Joint Monitoring Programme: WAT/SAN Categories, WHO/UNICEF, Geneva, Switzerland, 2015, http://www.wssinfo.org/definitionsmethods/watsan-categories/.

[26] N. Katz, A. Chaves, and J. Pellegrino, "A simple device for quantitative stool thick-smear technique in Schistosomiasis mansoni," Revista Instituto Medicina Tropical de Sao Paulo, vol. 14, no. 6, pp. 397-400, 1972.

[27] V. Khanna, K. Tilak, S. Rasheed, and C. Mukhopadhyay, "Identification and preservation of intestinal parasites using methylene blue-glycerol mount: a new approach to stool microscopy," Journal of Parasitology Research, vol. 2014, Article ID 672018, 4 pages, 2014.

[28] T. Acharya, "Direct saline/iodine wet mount for diagnosis of intestinal parasites (microbeonline website)," 2015, http:// microbeonline.com/saline-wet-mount-diagnosis-intestinalparasites/.

[29] E. A. Chadeka, S. Nagi, N. B. Cheruiyot et al., "A high-intensity cluster of Schistosoma mansoni infection around Mbita causeway, western Kenya: a confirmatory cross-sectional survey," Tropical Medicine and Health, vol. 47, no. 1, pp. 1-4, 2019.

[30] C. Mwandawiro, C. Okoyo, J. Kihara et al., "Results of a national school-based deworming programme on soiltransmitted helminths infections and schistosomiasis in Kenya: 2012-2017," Parasites \& Vectors, vol. 12, no. 1, 2019.

[31] J. H. Kihara, N. Muhoho, I. Mwobobia et al., "A four-year follow-up of school children after mass-treatment for schistosomiasis and soil transmitted helminths in Mwea, central Kenya," African Journal of Health Sciences, vol. 23, pp. 232237, 2012.

[32] World Health Organization, Vector Control-Methods for Use by Individuals and Communities (Chapter 8: Freshwater Snails), World Health Organization, and, 1997, https:/www.google. com/search?rlz=1C1GCEU_enIN894IN894\&q=Geneva\&stick= H4sIAAAAAAAAAOPgE-LQz9U3sDAxKFcCs4wtDYy0tLKTr fTzi9IT8zKrEksy8_NQOFYZqYkphaWJRSWpRcWLWNncU_ NSyxJ3sDICAOSE_QdOAAAA\&sa=X\&ved=2ahUKEwiImfrih YnpAhVX8XMBHTPfBhYQmxMoATApegQIChAD.

[33] P. M. Gichuki, S. Kepha, D. Mulewa et al., "Association between Schistosoma mansoni infection and access to improved water and sanitation facilities in Mwea, Kirinyaga County, Kenya," BMC Infectious Diseases, vol. 19, pp. 1-14, 2019.

[34] R. K. M’Bra, B. Kone, Y. G. Yapi et al., "Risk factors for schistosomiasis in an urban area in northern Côte d'Ivoire," Infectious Diseases of Poverty, vol. 7, no. 1, pp. 1-12, 2018.

[35] T. Handzel, A. W. Hightower, D. G. Colley et al., "Geographic distribution of schistosomiasis and soil-transmitted helminths in Western Kenya: implications for anthelminthic mass treatment," The American Journal of Tropical Medicine and Hygiene, vol. 69, no. 3, pp. 318-323, 2003.

[36] T. W. Ngonjo, J. H. Kihara, M. Gicheru, P. Wanzala, S. M. Njenga, and C. Mwandawiro, "Prevalence and intensity of intestinal parasites in school age children in Thika district,
Kenya," African Journal of Health Sciences, vol. 21, pp. 153160, 2012.

[37] E. K. Kamande, L. N. Muthami, and J. H. Ouma, "Prevalence and intensity of intestinal parasitic infections and factors associated with transmission among school going children," East African Medical Journal, vol. 92, pp. 264-269, 2015.

[38] B. Tulu, S. Taye, and E. Amsalu, "Prevalence and its associated risk factors of intestinal parasitic infections among Yadot primary school children of South Eastern Ethiopia: a crosssectional study," BMC Research Notes, vol. 7, no. 1, p. 848, 2014.

[39] S. S. W. Sakari, A. K. Mbugua, and G. M. Mkoji, "Prevalence of soil-transmitted helminthiases and schistosomiasis in preschool age children in Mwea division, Kirinyaga south district, Kirinyaga county, and their potential effect on physical growth," Journal of Tropical Medicine, vol. 2017, Article ID 1013802, 12 pages, 2017.

[40] J. E. T. Grimes, G. Tadesse, K. Mekete et al., "School water, sanitation, and hygiene, soil-transmitted helminths, and schistosomes: national mapping in Ethiopia," PLoS Neglected Tropical Diseases, vol. 10, pp. 1-21, 2016.

[41] L. C. Charts, M. M. Brooke, D. M. Melvin, G. R. Healy, and S. Edition, Common Intestinal Protozoa of Humans, U.S. Department of Health and Human Services, Atlanta, GA, USA, 1983.

[42] H. S. Mekonnen and D. T. Ekubagewargies, "Prevalence and factors associated with intestinal parasites among under-five children attending Woreta Health Center, Northwest Ethiopia," BMC Infectious Diseases, vol. 19, no. 1, pp. 1-8, 2019.

[43] T. Schmidlin, E. Hürlimann, K. D. Silué et al., "Effects of hygiene and defecation behavior on helminths and intestinal protozoa infections in Taabo, Côte d'Ivoire," PLoS One, vol. 8, Article ID e65722, 2013.

[44] J. Garn, B. Caruso, C. Drews-Botsch et al., "Factors associated with pupil toilet use in Kenyan primary schools," International Journal of Environmental Research and Public Health, vol. 11, no. 9, pp. 9694-9711, 2014.

[45] Ministry of Education, National School Water, Sanitation, and Hygiene Promotion Strategy: 2008-2015, Ministry of Education, Nairobi, Kenya, 2008.

[46] UNICEF, WASH in Schools (WinS) Country Profile Kenya, UNICEF, New York, NY, USA, 2011, http://washinschools mapping.com/wengine/wp-content/uploads/2015/10/Kenya WinsCountryProfile.pdf. 